\title{
Polizeikosten gegen Demonstranten
}

\section{Einleitung}

Noch vor einigen Jahren war es eine Selbstverständlichkeit, daß die Polizei für ihren Einsatz für die öffentliche Sicherheit und Ordnung in der Regel keine Kosten geltend machte. ${ }^{1}$ Inzwischen sind diejenigen, die sich für die Beibehaltung der grundsätzlichen Kostenfreiheit von Polizeieinsätzen stark machen, in der Defensive.

Von verschiedener Seite werden unterschiedliche Gründe für die Rechtsentwicklung angegeben. Die Kostenerstattung für Polizeieinsätze sei durch die leeren Kassen der öffentlichen Hände diktiert. ${ }^{2}$ Die Zahl von Veranstaltungen und die Tendenz zu Gewalttätigkeiten hierbei nähme zu. Zu deren Sicherung seien immer mehr Polizeikräfte nötig, was die Staatskasse nicht unerheblich belaste. ${ }^{3}$ Daß diese Belastungen durch die Allgemeinheit getragen werden müssen, sei nicht mehr einzusehen. Würtenberger spricht von einer Kommerzialisierung des "Wirtschaftsgutes « der öffentlichen Sicherheit. Deren Aufrechterhaltung sei längst nicht mehr eine nur dem Staat zustehende Aufgabe. Es habe sich ein privates Wach- und Sicherungsgewerbe entwickelt, das entgeltlich arbeitet. ${ }^{4}$ Dort wo die Wahrung der öffentlichen Sicherheit einen Marktwert hat, solle auch der Staat diesen geltend machen können.

Neben den privaten Großveranstaltungen, deren Polizeischutz gebührenpflichtig sein soll, stehen vor allem politische Demonstrationen im Blickfeld der Diskussion: Nach Auflösung einer Demonstration soli die Anwendung unmittelbaren Zwanges, d. h. die Räumung der Demonstranten, kostenpflichtig sein.

Der ehemalige baden-württembergische Innenminister und jetzige Verfassungsrichter Roman Herzog beklagte, es sei »höchst unbefriedigend, daß nach bisher geltendem Recht keine Möglichkeit besteht, Störer zum Ersatz von Kosten heranzuziehen, die dadurch entstehen, daß sie eine polizeiliche Verfügung nicht befolgen «. Der »beklagenswerte Rechtszustand « besteht inzwischen nicht mehr in Baden-Württemberg und Bayern (LVwVGKO v. 29. I I. 82, bwGBl. si 8; PolKV v. 2. 8. 83, bayGVBl. 555). Eine entsprechende Regelung in Niedersachsen (PolGO v. I4. I 2. 77, ndsGVBl. 647 i.d. F. y. 13. 7. 82, ndsGVBl. 285) wird inzwischen nach einem Urteil des OVG Lüneburg nicht mehr angewendet. ${ }^{6}$

Nach Ansicht Herzogs berühren die Polizeikosten-Ersatzregelungen in keiner Weise das in der Verfassung gewährleistete Recht auf friedliche Demonstration. ${ }^{7}$ Der

\footnotetext{
I Kühling, Kosten für den Einsatz der Polizei, DVB|. 198 I, 315 .

2 Broß, Zur Erstattung der Kosten von Einsätzen der Polizei, Verw. Archiv 1983, 388

3 Broß, Zur Erstattung der Kosten von Polizeieinsätzen, DVBl. 1983, 378.

4 Würtenberger, Erstattung von Polizeikosten, NVwZ $1983,193$.

SPressemitteilung des Innenministeriums Bad.-Württembg. Nr, 192/1982 vom 2. 12. 1982.

6 OVG Lüneburg NVwZ 1984, 323.

7 S. o. (Fußnote 5).
} 
Frage, ob dies zutrifft, soll unter anderem in dieser Untersuchung nachgegangen werden.

Auch wenn es offiziell nicht offen ausgesprochen wird, so ist nicht von der Hand zu weisen, daß dem Polizeikostenersatz, ähnlich einer Geldbuße, auch eine spezial- und generalpräventive Funktion zukommt. ${ }^{8}$ Nach Ansicht von Schilde soll mit diesem Mittel vor allem gewaltfreien Blockaden beigekommen werden, mit denen der Staat mit seinen bisherigen rechtlichen und politischen Mitteln nur schwer fertig wird. ${ }^{9}$

\section{Regelungen der Kostenpflicht für die Anwendung unmittelbaren Zwangs}

Nach der Räumung eines besetzten Hauses in der Arndtstraße in Hannover im Dezember 197 I wurden einem Hausbesetzer als Gesamtschuldner für Verpflegungs-, Reise-, Fahrzeugkosten usw., die bei der Räumung entstanden, 5459,22 DM abverlangt. In einem bahnbrechenden Urteil bestätigte das OVG Lüneburg diesen Bescheid in Höhe von 3470,42 DM. ${ }^{\circ} \mathrm{Als}$ Ermächtigungsgrundlage wurde das allgemeine niedersächsische Verwaltungskostengesetz anerkannt. Durch dieses Urteil bestärkt, erließ der Niedersächsische Innenminister am I 4. I 2. I 9777 eine Polizeigebührenordnung (ndsGVBl. S. 647), wo es lapidar heißt: »Für Amtshandlungen der Polizei auf dem Gebiet der Gefahrenabwehr und im Verwaltungszwangsverfahren, für Leistungen der Polizei, die nicht Amtshandlungen sind, sowie für die Benutzung polizeilicher Einrichtungen und Gegenstände werden Gebühren nach dem anliegenden Gebührenverzeichnis erhoben, wenn die Beteiligten als Pflichtige nach $\$ 6$ oder $\$ 7$ des ndsSOG oder auf Antrag dazu Anlaß gegeben haben«. Nach dem Gebührenverzeichnis kostete ein Polizeibeamter pro Stunde $38 \mathrm{DM}$, ein Diensthund s DM, ein Dienstpferd I 2 DM, ein PKW pro km 0,40 DM, ein WSP-Küstenboot über 3000 PS 440 DM pro Stunde, ein Luftfahrzeug für jede angefangene Viertelstunde 300 DM. Als nächstes Bundesland führte Baden-Württemberg eine ähnliche, aber differenziertere Regelung ein. Die Änderung der Vollstreckungskostenordnung vom 29. 11. I982 enthält neben Gebührenerhöhungen als wesentliche Regelung die pauschale Gebührenpflicht für die Anwendung unmittelbaren Zwanges. Bisher waren nur zwei Sonderfälle, die Sachwegnahme und die Zwangsräumung gebührenpflichtig $(\mathbb{S} 3,7, \mathrm{LVwVGKO}$ a. F.). Die Gebühr wurde auf $38 \mathrm{DM}$ pro Bediensteten und Stunde festgesetzt. Daneben sind noch eine Vielzahl von Auslagen erstattungsfähig $(\$ 8$ n. F.) Auf Pflichtige, die nicht Gesamtschuldner sind, können die Gebühren "angemessen verteilt « werden ( $\$ 9$ n. F.).

Als bisher letztes Bundesland legte Bayern in seiner Polizeikostenverordnung vom 2. 8.83 (bayGVB1. 555) die Gebührenpflicht jedes unmittelbaren Zwanges fest. Nach $₫$ I PolkV kostet die unmittelbare Ausführung einer Maßnahme, die Sicherstellung und die Anwendung unmittelbaren Zwanges ohne vorausgehenden Verwaltungsakt 20 bis $2000 \mathrm{DM}$, die Ausführung der Ersatzvornahme und unmittelbarer Zwang zur Durchsetzung eines vorausgehenden Verwaltungsaktes kostet 40 bis $200 \mathrm{DM}$, für andere Maßnahmen werden Gebühren bis zu I00 DM verlangt.

In Niedersachsen zog jetzt Innenminister Möcklinghoff alle bisher ergangenen

8 Würtenberger (Fußnote 4 ), S. 193.

9 Schilde, Die Demonstrationskostenverordnung ist rechtswidrig, in: Demonstration, Ungehorsam, Widerstand gegen Raketenstationierung, Vereinigung Berliner Strafverteidiger (Hrsg.) 1983, S. 86.

10 OVG Lüneburg NJW 1978, 721 . 
Rechnungen über Polizeieinsatzkosten zurück, nachdem das OVG Lüneburg die Leistungsbescheide wegen der Anti-Atom-Dorf-Räumung in Grohnde für ungesetzlich erklärt hat. ${ }^{11}$

\section{Praxis der Kostenerhebung bei politischen Demonstrationen}

Schleswig-Holstein: 24 Personen erhielten Leistungsbescheide über je 356,29 DM wegen einer Hausräumung in Elmshorn am I 5. 4. 8I. Das VG hob die Leistungsbescheide auf, da nicht nachweisbar war, daß die Besetzer die Räumungsaufforderung erhalten hätten. ${ }^{12}$ Für die Räumung des Anti-Atom-Dorfes in Brokdorf sollten die Bewohner I I 3000 DM bezahlen. ${ }^{13}$

Niedersachsen: Über die Leistungsbescheide wegen Räumung eines Hauses in der Arndt-Straße in Hannover, die das OVG im großen ganzen für rechtmäßig erklärte, wurde schon berichtet. Eingetrieben wurden von den 3470 DM nur 539,55 DM. Danach wurde die Sache nicht mehr ernsthaft betrieben. ${ }^{14}$

Wegen der Räumung des Anti-Atom-Dorfes Grohnde am 23.8. 77 ergingen am 3. 7. 79 Leistungsbescheide in Höhe von 212133 DM an ca. 2000 Leute. Das VG Hannover gab der Klage gegen die Bescheide über je 1060,66 DM statt. Das OVG Lüneburg bestätigte das Urteil am 25. 8. 83. Es fehlte an einer ausreichenden Gesetzesgrundlage. ${ }^{\text {Is }}$

Für die Räumung des besetzten Informationspavillons in Esensham wurden ca. 25000 DM gefordert. ${ }^{16}$

4I Bewohner des Anti-Atom-Hüttendorfes 1004 (Gorleben) erhielten wegen dessen Räumung Leistungsbescheide über je 1000 DM. Der Einsatz habe 2,5 Mio. DM gekostet, auf 2500 Demonstranten verteilt mache das 1000 DM pro Person. 37 Teilnehmer wurden zur Zahlung des Geldes verurteilt. Wegen der Räumung einer gewaltfreien Blockade des Bohrloches 1004 Frühjahr 1980 erhielten 40 Demonstranten Bescheide über insgesamt 2762728,25 DM.

Nach einer Hausräumung in Hannover am 24. 3. 8 $\mathbf{I}$ (Franz-Bork-Str. 8) erhielten II Leute Leistungsbescheide über insgesamt ca. $5000 \mathrm{DM}$. Weitere Räumungen in dieser Stadt am 14. 4. 8I (Velvestr. 6a) kosteten für 17 Leute insgesamt 2744 DM und am 11. 8. 81 (An der Stangenriede 39) für vier Personen insgesamt 447 DM.

Nach einer Hausräumung in Göttingen 198I erhielten 22 Personen eine Ankündigung von Leistungsbescheiden über insgesamt 36 I $82,40 \mathrm{DM}$. Und wegen der Räumung der Zufahrt des Baugeländes in Gorleben wurden 3 I Leistungsbescheide über insgesamt 2137,50 DM verschickt. ${ }^{17}$

Ein jüngerer Fall ist die Mastbesetzung in Grohnde, die den Demonstranten insgesamt fast 10000 DM kosten sollte.

4s DM wurden für Festnahme und Personalienfeststellung bei einer Walpurgisnachtdemonstration abgefordert. ${ }^{18}$

Baden-Württemberg: Die neue Vollstreckungskostenordnung trat am I I. I 2. 1982 in Kraft, gerade noch rechtzeitig, um 292 Teilnehmer an der Blockade der Befehlszen-

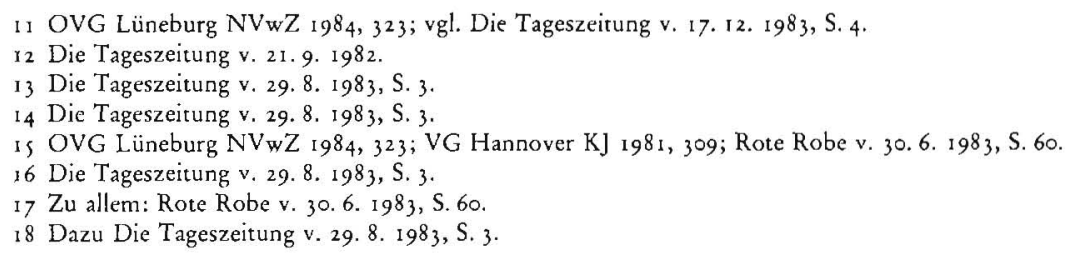


trale der US-Streitkräfte in Europa (EUCOM) am 1 2. I 2. 82 mit Leistungsbescheiden in Höhe von insgesamt 23000 DM zu belegen. Von den Blockierern wurden bis zu $210 \mathrm{DM}$ gefordert. ${ }^{19}$

Die Verordnung ist danach gegenüber Blockierern des Atomwaffenlagers »Golf « bei Großengstingen und des NATO-Flughafens in Lahr zur Anwendung gebracht worden. Im letztgenannten Fall sollen 38 Personen jeweils 47,30 DM bezahlen, also insgesamt ca. I $800 \mathrm{DM}$ von den $10556,88 \mathrm{DM}$, die als Kosten des Einsatzes veranschlagt wurden. ${ }^{20}$

Wegen der Besetzung des ehemaligen Pershing-Stützpunktes bei Inneringen am I6. I1. 83 verschickte die PD Sigmaringen am 20. 1. 8432 Kostenbescheide zur Deckung der Kosten der Räumung (7696 DM) von je 245,50 DM. ${ }^{21}$

Den polizeilichen Räumungen von Demonstranten vor dem Pershing-2-Standort folgten eine Vielzahl von Kostenbescheiden, z. B. kostete ein Einsatz am I 2. 9. 1983 für 26 Pflichtige insgesamt I $148,40 \mathrm{DM}^{22}$

Die Aufstellung der bisherigen Fälle zeigt, daß die Kostenerhebung wegen Anwendung unmittelbaren Zwanges im politischen Bereich einen Schwerpunkt hat. Mir ist nur ein Fall bekannt, in dem "einfache" Störer (ein sich streitendes Ehepaar) zu Gebühren von $25 \mathrm{DM}$ zur Kasse gebeten worden sind. ${ }^{23}$

\section{Verstoß gegen Grundrechte}

Hauptangriffspunkt gegen die neue baden-württembergische Kostenverordnung ist, daß die politischen Grundrechte der Versammlungs- und Meinungsäußerungsfreiheit nach Art. 5, 8 GG verletzt werden.

a) Die in Art. 8 I GG verankerte Demonstrationsfreiheit gewährleistet dem Bürger eine über Wahlen hinausgehende Einflußnahme auf den ständigen Prozeß der politischen Meinungs- und Willensbildung. Um die öffentliche Willensbildung beeinflussen zu können, muß dort demonstriert werden, wo der Öffentlichkeit die Gelegenheit gegeben ist, die demonstrierten Meinungen zur Kenntnis zu nehmen und zu diskutieren. Das führt zwangsläufig dazu, daß unbeteiligte Bürger, die mit der kollektiven Meinungskundgabe konfrontiert werden, in ihren Rechten und Interessen tangiert und auch beeinträchtigt werden. Diese Auswirkungen, die bei praktisch jeder Wahrnehmung der Demonstrationsfreiheit auftreten, können nicht pauschal als rechtswidrig angesehen werden. Selbst bewußte und gezielte Einwirkungen auf die Sphäre Dritter sind bei der Wahrnehmung des Demonstrationsrechts im geistig wirkenden Meinungskampf unvermeidbar. ${ }^{24}$ Auch der Einsatz des Körpers als Medium der Meinungsäußerung hält sich grundsätzlich im Rahmen der politischen Freiheitsrechte. ${ }^{25}$

Art. 8 GG schützt alle friedlichen Versammlungen deutscher Staatsbürger in Räumen und unter freiem Himmel. Eine Demonstration wird nicht schon deshalb unfriedlich, weil sie mit Rechten unbeteiligter Dritter kollidiert. Dies wäre nur dann der Fall, wenn der Verletzungs- und Schädigungswille im Vordergrund steht. ${ }^{26}$ Eine

19 Rote Robe v. 30. 6. 1983 , S. 60; Frankfurter Rundschau v. 2. 2. 1983.

20 Badische Zeitung v. 1.9. 1983.

21 Die Tageszeitung v. 24, I, I984, S. I.

22 Bescheide hat der Autor.

23 Die Tageszeitung v. 29.8 . 1983 , S. 3.

24 Vgl. BVerfGE 25, 256 - Vereinbarkeit eines Boykottaufrufs mit Art. s I I GG.

25 Erichsen, Staatsrecht und Verfassungsgerichtsbarkeit, 2. Aufl. 1979, S. 163.

26 AG Stuttgart KJ $1983,435$. 
Versammlung ist dann nicht mehr friedlich, wenn ein "gewalttätiger oder aufrührerischer Verlauf « angestrebt ist oder erfolgt (vgl. $\mathbb{S} 5$ S Ziff. 3, 13 I Ziff. 2 VersG).

Ein bloßer Sitzstreik ist niemals Gewalt im Sinne des Versammlungsrechts, sondern ein Musterbeispiel gewaltloser Handlungsweise, auch wenn dadurch Dritte behindert werden. ${ }^{27}$ Gleiches gilt für andere "gewaltfreie Aktionen «, wie »Mahnwachen «, "Menschenteppiche« oder »Menschenketten«. Die nicht selten zu findende Ansicht, Sitzblockaden fielen nicht unter den Schutzbereich des Art. 8 GG, ist nicht haltbar. Danach soll Art. 8 GG kein Recht auf Handlungen gewähren, die über das hinausgehen, was einem Einzelnen erlaubt ist. Damit verlöre Art. 8 jeglichen Gehalt als kollektives Grundrecht und gewährleistete nur Meinungsäußerungen, die auch auf Grund einfachgesetzlicher Regelung zulässig sind. Es geht nicht an, daß die ausdehnende Rechtsprechung zum Gewaltbegriff im Strafrecht ( $\$ \$ 124,125,240 \mathrm{StGB}$ ) auf die Auslegung der "Unfriedlichkeit» bei Art. 8 GG übertragen wird ${ }^{28}$ Denn damit würde das Grundgesetz als höherrangiges Gesetz nach Maßgabe des StGB als eines einfachen Gesetzes interpretiert. ${ }^{29}$

Die neue Kostenverordnung setzt Gebühren fest, wenn Störer nach Polizeirecht eine gegen sie ergangene polizeiliche Verfügung nicht befolgen und dadurch die Androhung und Anwendung unmittelbaren Zwangs nach $\$ 26 \mathrm{LVwVG}$ nötig machen

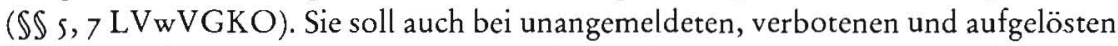
Demonstrationen angewendet werden. Sie wird angewendet bei Blockaden, Sitzstreiks und ähnlichen "gewaltfreien Aktionen «, wenn die Teilnehmer die Verfügung, sich zu entfernen, nicht befolgen.

Verbot, Auflösung und deren Durchsetzung mit unmittelbarem Zwang halten sich noch im Rahmen des Art. 8 II GG. Diese Grundrechtsbeschränkungen sind zulässig, ihre Folgen sind für die Teilnehmer erträglich, begrenzt und absehbar. Bei den generalklauselartigen Begriffen, die die Zulässigkeit einer Versammlungsauflösung umschreiben, ist aber eine eindeutige Feststellung der Rechtmäßigkeit der konkreten Auflösungsverfügung nicht möglich. Dies gilt z. B. dann, wenn zur Abwehr einer Gefahr für die öffentliche Sicherheit schon eine Auflage als schwächeres Mittel ausreicht.

Die Grenze zwischen zulässiger Demonstration und rechtswidriger Störung sind fließend. Eine eindeutige Festlegung ist nur durch die Gerichte möglich. Auf jeden Fall darf die Entscheidung über die Zulässigkeit eines Beitrags im politischen Meinungskampf nicht im wesentlichen der Polizei, konkret einem Verwaltungsbeamten oder Einsatzleiter, überlassen bleiben. Sind an die Entscheidung des Verwaltungsbeamten oder Einsatzleiters Folgen geknüpft, die einen Bürger grundsätzlich von der Wahrnehmung eines grundrechtlichen Demonstrationsrechts abhalten, so wird unzulässig in dieses Grundrecht eingegriffen. Ist mit der Anwendung unmittelbaren Zwanges zur Durchsetzung einer Verbots- oder Auflösungsverfügung durch die Polizei eine Gebührenpflicht verbunden, so liegt darin ein über die Räumung hinausgehender Grundrechtseingriff. Teilt ein Demonstrant nicht die Ansicht der Polizei über die Rechtswidrigkeit einer Versammlung und bleibt er trotz polizeilicher Verfügung am Versammlungsort, so läuft er Gefahr, wegen Polizeikosten herange-

27 Klug, Strafrechtliche Probleme des Demonstrationsrechts, in: Demonstrationsfreiheit, Strafrecht und Staatsgewalt, Frankfurt 1968; Ott, Gesetz über Versammlungen und Aufzüge, 4. Aufl. 1983, Anm. 11 zu $₫ 5$; Herzog, in: Maunz/Dürig/Herzog/Scholz, Kommentar zum GG, 21. Lfg., Rdn. 62 Fußn. 6 zu Art. 8; v. Brünneck, Die Kostenerhebung der Polizei bei Demonstrationen, NVwZ 1984, 275; vg]. OVG Lüneburg NVwZ 1984, 323.

28 Ott (Fußnote 27) $\$ 5$ Rdn. 11 .

29 Erichsen (Fußnote 25), S. 162 
zogen zu werden. Diese Gefahr kann ihn auch vom rechtmäßigen Verbleiben in der Versammlung abhalten, wenn sich nämlich die Auflösungsverfügung als rechtswidrig herausstellt; zugleich ist er damit in seinem Grundrecht beschnitten. ${ }^{30}$

Dem einzelnen Demonstranten dürfen keine Risiken aufgebürdet werden, die er wegen der geringen Bestimmtheit der Generalklausel des $\$$ is VersG nicht überblikken kann.

b) Es ist prinzipiell davon auszugehen, daß für polizeiliche Einsätze in Verbindung mit politischen, religiösen und wissenschaftlichen Veranstaltungen keine Kostenerstattung verlangt werden kann. ${ }^{31}$

Bei der baden-württembergischen LVwVGKO ist die Gebührenhöhe gekoppelt an Zahl und Einsatzdauer der Polizeibeamten und an den materiellen Aufwand des Polizeieinsatzes $(\$ \$ 7,8 \mathrm{LVwVGKO})$. Sie hängt von der Ermessensentscheidung des polizeilichen Einsatzleiters ab und ist für den Versammlungsteilnehmer unabsehbar, grundsätzlich unbegrenzt und unverhältnismäßig. ${ }^{32}$

Dieses Problem läßt sich nicht dadurch aus der Welt schaffen, indem verlangt wird, daß die Polizei den potentiellen und tatsächlichen Störern über das Ausmaß der zu erwartenden Kosten Klarheit verschafft. ${ }^{33}$ Anders als bei Großveranstaltungen ist ein solches Vorgehen nicht praktikabel. Die Kostenhöhe läßt sich erst mit Eintritt der Störung abschätzen. Dieser Zeitpunkt fällt praktisch mit dem Beginn der Gebührenpflicht zusammen.

Gegen die grundsätzliche Unbegrenztheit und Unabsehbarkeit der Kosten läßt sich nicht vorbringen, daß die Kostenerhebung auch bezüglich der Erforderlichkeit des Einsatzes voll nachprüfbar sei. ${ }^{34}$ Die nachträgliche Überprüfbarkeit durch die Gerichte ändert nichts an der Unvorhersehbarkeit der Kostenhöhe zur Zeit des Einsatzes. Äußerst umstritten ist auch, ob die Frage der Erforderlichkeit des Einsatzes bei der Kostenerhebung voll überprüfbar ist.3s Davon wäre grundsätzlich auszugehen, da der Grundrechtseingriff durch die Gebührenerhebung nicht dem freien Ermessen einer Behörde überlassen sein darf. Parallelen zu Prognose- und Planungsentscheidungen sind bei der Rechtmäßigkeitsüberprüfung der Kosten nicht angebracht. ${ }^{36}$

c) Die Kostendrohung in unbestimmter Höhe wirkt wic ein selbständiges, empfindliches und vorbeugend abschreckendes Zwangsmittel. ${ }^{37}$ Durch diese präventive Wirkung der Gebührenpflicht verliert sie den repressiven Charakter der Verwaltungsvollstreckung und wird zu einer präventiven strafähnlichen Geldbuße. ${ }^{38} \mathrm{Das}$ Risiko, wegen der Teilnahme an einer Demonstration zu Kosten herangezogen zu werden, ist durchaus geeignet, Personen, die von der Rechtmäßigkeit ihres Demonstrierens überzeugt sind, hiervon abzuhalten. ${ }^{39}$

Schenke rechtfertigt die disziplinierende Wirkung der neuen Kostenverordnung.

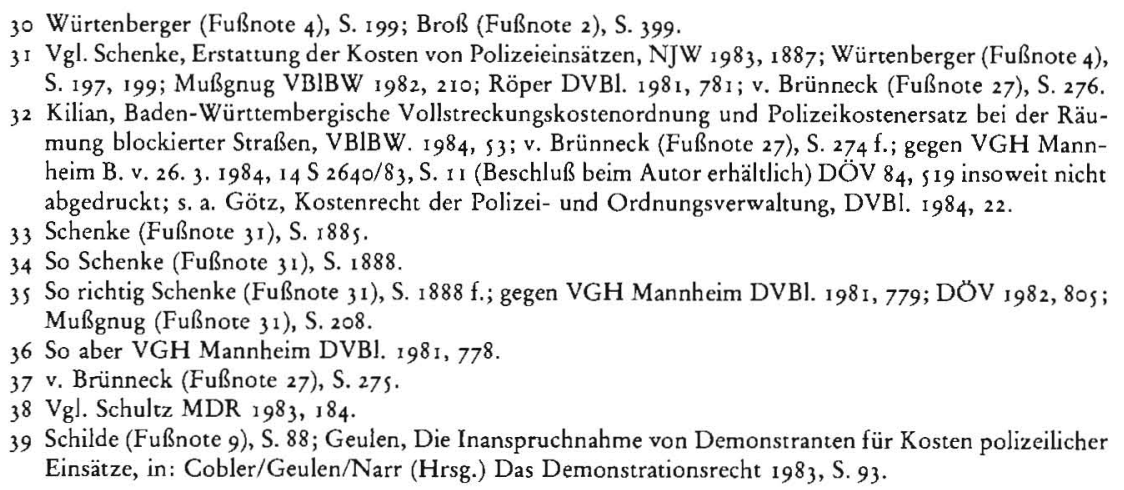


"Lenkende Gebühren «, bei denen mit der Erhebung die Disziplinierung des Schuldners bezweckt würden, seien ein rechtlich längst anerkanntes Instrument, das sich problemlos in das Vollstreckungsrecht einpasse..$^{40} \mathrm{Dem}$ ist z.war grundsätzlich zuzustimmen. Etwas anderes gilt aber, wenn mit der Regelung des Polizeikostenersatzes die Tendenz verfolgt wird, die Ausübung der durch das Versammlungsgesetz garantierten Freiheiten wesentlich zu erschweren. ${ }^{41}$

Der abschreckende Effekt der Kostenerhebung ist nicht nur eine erwünschte Nebenfolge der staatlichen Finanzbeschaffung, sondern der vorrangig verfolgte $Z$ weck. Im Runderlaß zur niedersächsischen Kostenverordnung hieß es: »... sollten die Störer nach Möglichkeit auf die Kostenfolge hingewiesen werden. Hierdurch könnte eine zusätzliche präventive Wirkung erzielt werden « ${ }^{42}$ In Baden-Württemberg ist die Lage noch eindeutiger: Die neue LVwVGKO vom 29.11. 82 trat am II. 12. 82 in Kraft, gerade noch rechtzeitig, um am I2.12. 1982 gegen Demonstranten vor dem US-Hauptquartier in Stuttgart-Vaihingen angewendet werden zu können. Die Verordnung war eine direkte Reaktion auf die gewaltfreien Blockaden in Baden-Württemberg und zielt weniger auf Finanzbeschaffung, sondern vor allem auf Abschrekkung ab. Ministerpräsident Späth: "Wenn das der große Trick war mit dem Wegtragen lassen, dann war das mit unserer Gebührenordnung halt auch so eine Gegenmaßnahme - jetzt müßt ihr euch etwas Neues einfallen lassen $\propto{ }^{43}$ Der damalige Innenminister Herzog: »Für mich steht das Finanzielle nicht im Vordergrund... Ich verkenne natürlich nicht, daß sich wirtschaftliche Sanktionen für den Adressaten genauso entscheidend auswirken wie meinetwegen der Schlagstock «. ${ }^{44} \mathrm{Da}$ mit der neuen LVwVGKO die Tendenz verfolgt wird, die Ausübung von gewaltfreien Blokkaden, Sitzstreiks usw., welche durch Art. 8 GG geschützt sind, wesentlich zu erschweren, liegt ein Verstoß gegen das Grundrecht der Versammlungsfreiheit vor.

d) Der abschreckende, von der Wahrnehmung des Rechts auf Versammlungsfreiheit abhaltende Charakter der Kostenverordnung wird dadurch verstärkt, daß die Gebührenpflicht auch dann besteht, wenn die auferlegte Pflicht erfüllt wird, nachdem sich die Polizeibediensteten zur Anwendung des unmittelbaren $Z$ wanges an Ort und Stelle begeben haben ( $\$ 7$ I 2 LVwVGKO). Mit dieser Regelung erhöht sich die Ungewißheit für die Versammlungsteilnehmer, von welchem Zeitpunkt an sie kostenpflichtig werden.

In der Regel befinden sich die Polizeibeamten schon zu Beginn der Demonstration an Ort und Stelle. Die Kostenpflicht wird so unmittelbar nach der polizeilichen Verfügung begründet. Den Demonstranten trifft die Kostenpflicht, ganz gleich, ob er sich aus der Demonstration entfernt oder nicht. Er wird zu Kosten herangezogen, die er überhaupt nicht verursacht hat. ${ }^{45}$ Es gibt für ihn auch keinen materiellen Grund, der Polizeiverfügung zu entsprechen - er muß für den Polizeieinsatz bezahlen, unabhängig davon, ob gegen ihn tatsächlich unmittelbarer $Z_{\text {wang angewendet }}$ wird. $4^{6}$

Bisher war allgemein anerkannt, daß Voraussetzung für das Verwaltungszwangsverfahren die Nichtbefolgung des Verwaltungsakts ist. ${ }^{47}$ Dieser Grundsatz soll nicht mehr gelten. Diese Vorverlagerung der Kostenpflicht ist nicht nur ein schwerwie-

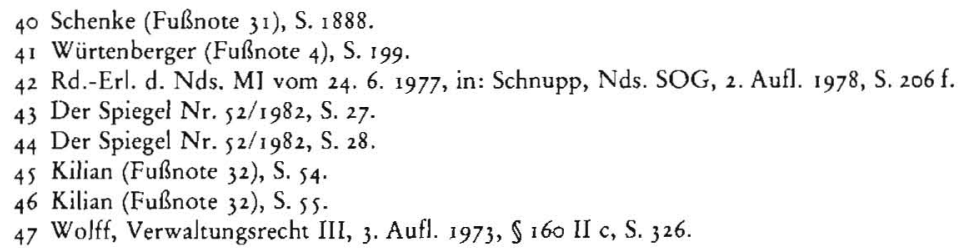


gender Verstoß gegen Art. 8 GG, sondern verletzt auch das in Art. 20 III GG garantierte Rechtsstaatsprinzip.

e) Die Anwendung der LVwVGKO ist ein Eingriff in die Versammlungsfreiheit, der nur durch eine gesetzliche Regelung zulässig wäre. Bei einer solchen Regelung nach Art. 8 II GG müßte das Zitiergebot des Art. 19 I 2 GG beachtet werden. Als Ermächtigungsgrundlage für die Kostenverordnung kommen nur das LVwVG oder das Polizeigesetz in Frage. $\$ 29$ LVwVG nennt ebenso wenig wie $\$ 4$ PolG die Versammlungsfreiheit als durch das Gesetz jeweils eingeschränktes Grundrecht. Die Zitierung wäre unbedingt geboten gewesen..$^{8}$

f) Das Ausmaß der Kostenerstattungspflicht kann existenzvernichtende oder -bedrohende Wirkungen auf die Pflichtigen haben.49

Die Schutzwirkung des Art. ${ }_{14}$ GG verbietet die Kostenerhebung, wenn die Gebührenhöhe den Schuldner in seinen Vermögensverhältnissen beeinträchtigt oder gar zu dessen Existenzvernichtung führt. ${ }^{5 \circ}$ Darin liegt zugleich eine Verletzung des Wesensgehalts des Grundrechts der Versammlungsfreiheit i. S. v. Art. I9 II GG. Solche Folgen werden vor allem bei gesamtschuldnerischer Haftung der Demonstrationsteilnehmer eintreten. ${ }^{51} \mathrm{Nach} \$ 9$ I LVwVGKO werden die Kosten in den Fällen des $\$ 7$ LVwVGKO auf die beteiligten Pflichtigen »angemessen verteilt«, aber nur gegen Pflichtige, die nicht Gesamtschuldner sind. In der Anwendung der badenwürttembergischen Kostenverordnung wurde bisher immer eine »angemessene Verteilung « praktiziert. Nach dem Wortlaut des $\$ 9$ und der Rechtsprechung zur Gesamtschuld wäre aber jedes Mal die Auferlegung aller Kosten auf einen gesamtschuldnerisch haftenden Pflichtigen möglich gewesen. Nach ständiger Rechtsprechung des BGH sind bei einer Demonstration i. d. R. alle Demonstranten als Mittäter anzusehen, so daß jeden die volle Haftung für Demonstrationsschäden trifft. ${ }^{52}$ Mithaftung wird selbst auf den Fall der bloß psychischen Unterstützung erstreckt. ${ }^{53}$ Zwar reicht die bloße Teilnahme an der Demonstration nicht aus, es genügt aber, daß der Wille der Demonstrationsteilnahme auch die aus ihr hervorgegangenen Handlungen umfaßt. ${ }^{54}$ Diese weitgehende Rechtsprechung wurde neuerdings vom BGH teilweise zurückgenommen. Danach ist für die Gesamtschuldnerschaft eine zeitliche und räumliche Nähe nötig sowie ein deutlicher Tatbeitrag des Pflichtigen."s Legt man auch die neue Rechtsprechung des BGH zugrunde, so haften Teilnehmer an einer Sitzblockade immer als Gesamtschuldner. ${ }^{56}$

In $\$ 9$ I LVwVGKO ist die Gesamtschuld nicht nur Rechtsfolge, sondern Tatbestandsvoraussetzung, d.h. es muß Gesamtschuld bezüglich der Ordnungspflicht bestehen, um eine gesamtschuldnerische Haftung wegen der Polizeikosten zu begründen.

Der Verordnungsgeber kann bei der Gesamtschuldregelung nur von der Anwendbarkeit der zivilrechtlichen Grundsätze auch im Polizeirecht ausgegangen sein. Folgte man nämlich der Ansicht Seiberts ${ }^{57}$, wonach polizeirechtliche Gesamtschuld bei höchstpersönlichen Ordnungspflichten nicht möglich ist, so wäre auch keine

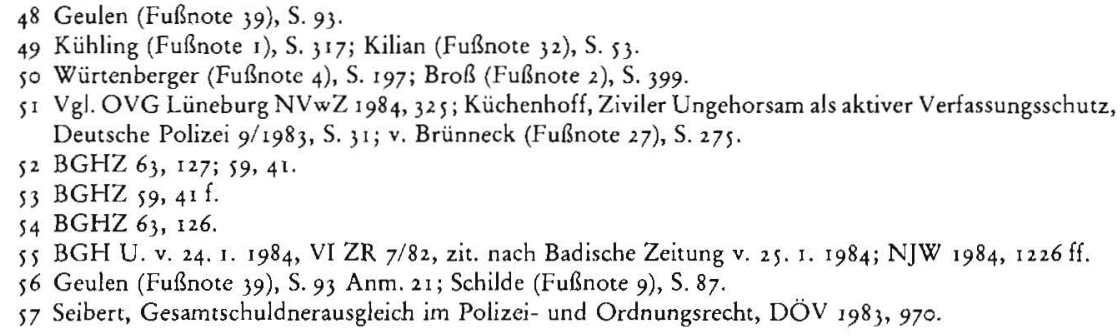


angemessene Verteilung bei fehlender gesamtschuldnerischer Haftung denkbar. Die Regelung des $\$ 9$ I Nr. 2 LVwVGKO würde unsinnig, da sich der kostenpflichtige Vollstreckungsakt dann nur auf den einzelnen Störer und nicht auf eine Mehrzahl von Störern beziehen kann. ${ }^{58}$

Geulen hat darauf hingewiesen, daß anders als bei Verbot und Auflösung einer Versammlung nach $\$$ is Vers $G$, die mit einmaligem Vollzug ihre Wirkung verlieren, die Kostenpflicht nach der LVwVGKO den Teilnehmer für einen längeren Zeitraum, gegebenenfalls für das gesamte Leben, treffen kann. Auch die Familie, insbesondere Unterhaltsberechtigte, können betroffen sein. 59

Die Anwendung der Kostenverordnung in Baden-Württemberg brachte bisher Kostenbescheide in Höhe von 47,30 DM (Blockade in Lahr, 27. 5. 83) bis zu 245,50 DM (Militärplatzbesetzung Inneringen, I6. I I. 83). Die Gesamtkosten der Einsätze betrugen $7696 \mathrm{DM}$ (Inneringen), 10 556,88 DM (Lahr), durch Kostenbescheide an die EUCOM Blockierer in Stuttgart am I 2. 1 2.82 sollten 20-25000 DM eingebracht werden. ${ }^{60}$ In Niedersachsen, wo eine der LVwVGKO ähnliche Berechnungsgrundlage galt (Nds.PolGVO v. I3. 7. 82, GVBl. 28 s; zuvor vom 14. 12. 77 GVBl. 647), wurden u. a. folgende Kosten erhoben: Räumung des Anti-AtomDorfes Grohnde am 23. 8. 77: 2 I 2 I 33 DM, Räumung des Anti-Atom-Dorfes 1004 (Gorleben) Frühjahr $19802762728,25 \mathrm{DM}^{62}$

Selbst die angemessene Verteilung der - exemplarisch dargestellten - Kosten kann Demonstranten so existentiell treffen, daß deren Grundrechte nach Art. 8 I, I4 GG im Kernbereich beeinträchtigt sind (Art. I9 II GG).

g) Der Kernbereich des Art. 8 I GG wird zudem dadurch tangiert, daß die pflichtigen Demonstranten bei der gesamtschuldnerischen Haftung auf den Rückgriff gegen andere Demonstrationsteilnehmer verwiesen werden. ${ }^{62}$ Dies führt in der Konsequenz zu einer Vielzahl von zivilen Rechtsstreitigkeiten von Demonstrationsteilnehmern untereinander. Dieser prozessuale Zwang zur Desolidarisierung und Isolierung der Demonstranten untereinander trifft gerade die kollektive demokratische Seite der in Art. 8 gewährleisteten Demonstrationsfreiheit. ${ }^{63}$

h) Eine Demonstration stellt grundsätzlich auch eine Meinungsäußerung der Versammlungsleitung und der Teilnehmer dar. Diese konkludente kollektive Meinungsäußerung steht unter dem Schutz des Art. s GG. Art. 8 GG ist hierzu Spezialvorschrift. Auch die Einschränkung der Freiheit auf Meinungsäußerung bedarf eines allgemeinen Gesetzes und ist durch Rechtsverordnung unzulässig. Art. 5 GG bietet allerdings keinen über Art. 8 GG hinausgehenden Schutz. ${ }^{64}$

\section{Vereinbarkeit mit weiteren Verfassungsgrundsätzen}

a) In der LVwVGKO ist die Inanspruchnahme für Kosten polizeilicher Einsätze vorgesehen. Diese Inanspruchnahme ist nach überzeugender Ansicht in der juristischen Literatur rechtlich grundsätzlich ausgeschlossen. ${ }^{65}$ Kostenerhebung für Poli-

\footnotetext{
s8 Die Frage der Gesamtschuld kann nicht weiter vertieft werden, vgl. dazu Seibert (Fußnote s7), S. 964 ff., inbes. S. 970 .

59 Geulen (Fußnote 39), S. 94.

60 Herzog, in: Der Spiegel Nr. \{2/82, S. 28

61 Die Tageszeitung v. 29.8. 1983, S. 3 .

62 Seibert (Fußnote 57), S. 974; Blanke/Sterzel, in: Das Demonstrationsrecht (Fußnote 39), S. 76.

63 Geulen (Fußnote 39), S. 94 m. V. a. Herzog (Fußnote 27), Art. 8, Rdn. 1-8.

64 Herzog (Fußnote 27), Art. 8 Rdn. 29.

6s Albrecht, Probleme der Kostenerhebung für polizeiliche Maßnahmen, in: Schreiber (Hrsg.), Festschrift

für Rudolf Samper, 1982, S. 176 ff.; so wohl auch Kilian (Fußnote 32), S. s4.
} 
zeieinsätze ist in der Geschichte des Polizeirechts ein Novum. Früher wurden Polizei- und Ordnungsbehörden i.d. R. kostenfrei tätig. ${ }^{66} \mathrm{Daß}$ dies auch so bleiben muß, ergibt eine Analyse des Steuer- und des Gebührenbegriffs. Gebühren werden für die besondere Inanspruchnahme oder Leistung der öffentlichen Verwaltung im Einzelfall verlangt, während Steuern für unverzichtbare allgemein notwendige Staatstätigkeiten erhoben werden. Die Tätigkeit der Polizei mit dem Ziel der Bewahrung von Sicherheit und Ordnung gehört dem Kern staatlichen Handelns an. Sie ist nach der Theorie des Gesellschaftsvertrags die Gegenleistung für Gehorsam und Finanzierung durch die Bürger. Begreift man Steuern als Gegenleistung ohne besondere individualisierbare Gegenleistung an den einzelnen Bürger ${ }^{67}$, so kann davon ausgegangen werden, daß mit diesen Steuern die grundlegendsten Staatsaufgaben finanziert werden. Daraus folgert Albrecht, daß der Bürger für die polizeiliche Tätigkeit, die bereits durch Besteuerung gedeckt ist, nicht nochmals zur Kasse gebeten werden darf. Eine solche Doppeldeckung widerspricht dem Rechtsstaatsprinzip. $^{68}$

Wenn dem entgegengehalten wird, Steuern seien nicht zweckgebunden, es obliege der freien Entscheidung des Gesetzgebers, wie er staatliche Tätigkeit finanziere, so ist dem grundsätzlich nicht zu widersprechen. ${ }^{68 \mathrm{a}}$ Das Rechtsstaatsprinzip verbietet aber auf jeden Fall dort eine Kostenerhebung, wo ganz vorwiegend öffentliche Interessen oder für den Rechtsstaat konstituierende Grundrechte tangiert sind. Nicht mehr zu vertreten ist die Kostenerhebung in dem für unsere Demokratie besonders empfindlichen Grundrechtsbereich der Meinungs- und Versammlungsfreiheit.

b) Die LVwVGKO verstößt gegen das aus dem Rechtsstaatsprinzip abgeleitete Verhältnismäßigkeitsprinzip. Nach ständiger Rechtsprechung des BVerfG hat der Gesetzgeber bei Festlegung von Kosten und Gebühren einen weiten Gestaltungsspielraum. Im Hinblick auf die Auswirkungen von Gebühren auf die Wahrnehmung von Grundrechten führte das BVerfG aber klarstellend aus: „Allgemeine Grenzen ergeben sich insbesondere aus dem Gleichheitssatz des Art. 3 Abs. I GG sowie aus dem Grundsatz der Verhältnismäßigkeit (im engeren Sinne), demzufolge die mit der Gebührenregelung verfolgten $Z$ wecke nicht außer Verhältnis zu der dem Bürger auferlegten Gebühr stehen dürfen. Dabei sind alle mit einer Gebührenregelung verfolgten verfassungsrechtlich zulässigen Zwecke als Abwägungsfaktoren in die Verhältnismäßigkeitsbetrachtung einzubeziehen. «69

Bei der Abwägung des Eingriffs durch die Leistungsanforderung gegenüber dem Bürger, insbesondere dem durch Art. 8 GG geschützten Bürger, und den durch die LVwVGKO verfolgten und erreichten $Z$ wecken ist folgendes mit zu berücksichtigen: Mit der Änderung der LVwVGKO waren auch fiskalische Überlegungen verbunden. Doch wurden diese selbst vom Verordnungsgeber nicht für ausschlaggebend betrachtet. ${ }^{70}$ Nach einjähriger Anwendung der neuen Kostenverordnung stellt die Polizeigewerkschaft fest, daß deren Anwendung insbesondere bei Demonstrationen einen Verwaltungsaufwand bedeute, der durch die eingezogenen Gebühren in keinem Verhältnis stünde. Insbesondere belaste die Beweisführung für die Kostenbescheide den Vollzugsdienst. ${ }^{71}$ Außerdem macht das Eintreiben der Kosten große

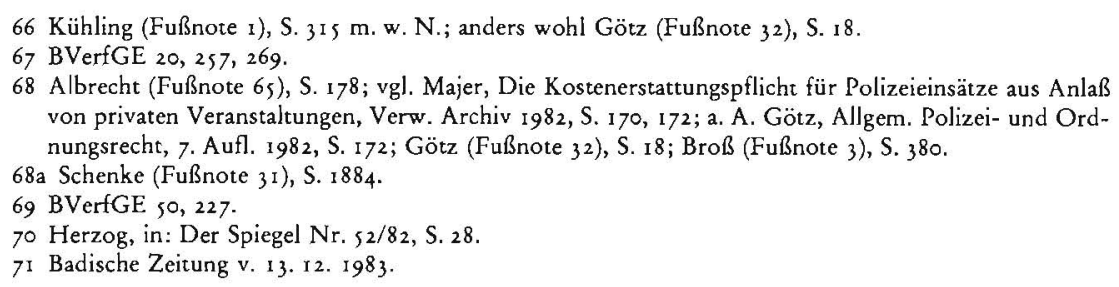


Schwierigkeiten wegen des geringen Vermögens und Einkommens vieler Pflichtiger und wegen deren geringer Zahlungsbereitschaft. So konnten z. B. von den 3470 DM Einsatzkosten für eine Hausräumung in Hannover I97 I nur 539,55 DM eingetrieben werden. ${ }^{72}$ Unberechtigt war die Hoffnung des Verordnungsgebers, daß die Gebührenerhebung bei Demonstrationen bestimmte Teilnehmer abhalten werde. Seit Inkrafttreten der neuen Kostenordnung haben gerade die Demonstrationsformen besonders zugenommen, gegen welche sich die Neuregelung richtet. Im Jahre 1983 erlebten Sitzblockaden, Mahnwachen, Menschenketten usw. geradezu einen Boom.

Hält man hiergegen die Beeinträchtigungen der kollektiven und individuellen Meinungs- und Versammlungsfreiheit, die oben dargestellte Möglichkeit großer finanzieller Belastungen für die Pflichtigen und die geringen Voraussetzungen zur Begründung der Kostenpflicht (die Kostenpflicht kann durch eine Störung ausgelöst werden, die nicht einmal den Tatbestand einer Ordnungswidrigkeit erfüllt), so liegt ein Verstoß gegen den Verhältnismäßigkeitsgrundsatz und gegen das Übermaßverbot auf der Hand. Weiterhin wird von verschiedener Seite darauf hingewiesen, daß auch die Zielrichtung von Sitzblockaden und ähnlichen Aktionen zu berücksichtigen seien. Das uneigennützige Sich-Einsetzen für das kollektive Überleben als hochrangiges Demonstrationsziel darf bei der Verhältnismäßigkeitsprüfung nicht unbeachtet bleiben. ${ }^{73}$ Der Zweck des Polizeikostenbescheids steht außer Verhältnis zur dem Bürger auferlegten Gebühr. ${ }^{74}$

c) In der Literatur bisher nicht diskutiert wurde im Zusammenhang mit der Erhebung von Polizeieinsatzgebühren ein Verstoß gegen das Verbot der Doppelbestrafung. Nach Art. IO$_{3}$ III GG darf niemand wegen derselben Tat auf Grund der allgemeinen Strafgesetze mehrmals bestraft werden. Durch diese Vorschrift soll neben der mehrmaligen Bestrafung auch die Belastung des Bürgers durch mehrere Strafverfahren verhindert werden. ${ }^{75}$ Wird mit einer Sanktion derselbe Zweck verfolgt und dieselbe Form gewählt, so gilt bei der gleichen Tat das Verbot der Doppelbestrafung, selbst wenn diese zweite Sanktion nicht dem allgemeinen Strafrecht angehört. ${ }^{76}$

Mit der neuen LVwVGKO wurden vor allem general- und spezialpräventive Zwecke verfolgt. Diese Funktionen sind die ureigensten Aufgaben des Straf- und Ordnungswidrigkeitenrechts. Fiskalische Gesichtspunkte spielten dagegen keine ausschlaggebende Rolle. Die Form der Übelzufügung der LVwVGKO entspricht der der Geldstrafe bzw. -buße. Ihre Höhe kann erheblich über den von Strafgerichten verhängten Geldstrafen liegen. Die zusätzliche Erhebung von Polizeikosten zwingt den Betroffenen ein weiteres (Verwaltungs-) Verfahren auf und belastet ihn mit einer weiteren Geldforderung, deren Funktion der einer Kriminalstrafe gleicht. ${ }^{77}$

\footnotetext{
72 Die Tageszeitung v. 29. 8. 1983.

73 Küchenhoff (Fußnote SI), S. 27; Erichsen (Fußnote 25), S. 161 m. w. N.

74 Geulen (Fußnote 39), S. 91 f.; Klug, NDR-Juristenstammtisch über Demonstrationskostenrecht, Protokoll Ende 1983 , S. Is.

75 Dürig, in: Maunz/Dürig/Herzog/Scholz (Fußnote 27), Art. I03 III Rdn. I 28.

76 Hamann, in: Hamann/Lenz, Grundgesetzkommentar, 35. Aufl. 1970, Art. 103 Anm. 5.

77 So wohl auch v. Brünneck, NDR-Juristenstammtisch (Fußnote 74), S. I sa.
} 
Die Polizei wird bei der Anwendung unmittelbaren Zwanges während Demonstrationen in der Regel sowohl präventiv-polizeilich als auch als Strafverfolgungsbehörde aktiv. Hieraus ergeben sich erhebliche Rechtsprobleme.

a) Die Kostenregelung nach Strafverfahrensrecht weicht von der polizeirechtichen stark ab. Dadurch entstehen Ungereimtheiten, die einen Verstoß gegen Art. 3 GG mit sich bringen. Im Strafverfahren sind dem Angeklagten Gebühren für polizeiliche Tätigkeit als Kosten des Ermittlungsverfahrens gemäß $\$ 4_{4} 6_{4}$ a StPO erst nach der Verurteilung aufzuerlegen. ${ }^{7}$ Diese Gebühren betragen maximal 300 DM. Der neue $\$ 7 \mathrm{LVwVGKO}$ wurde bisher nur in Fällen angewendet, die beim parallel durchgeführten Strafverfahren zu Geldstrafen von maximal 90 Tagessätzen führten. Für solche Verfahren entsteht eine Gerichtsgebühr von nur so DM (Anlage I zum GKG, Nr. I600; $\$ 40$ GKG). Die zusätzlich vom Angeklagten zu erstattenden Auslagen sind in sachlicher Hinsicht begrenzt und beinhalten nicht die Reisekosten der eingesetzten Beamten, die aber nach $\$ 8 \mathrm{I}$ Nr. $5,6 \mathrm{LVwVGKO}$ ersetzt werden müssen (Anlage zum GKG, Nr. $1900 \mathrm{ff}$.). Es werden also für gleiche Amtshandlungen sowohl Strafverfahrens- als auch Vollstreckungsgebühren verlangt, wobei in den meisten Fällen die Strafverfahrenskosten niedriger sind.

Eine Unterscheidung zwischen den Gebühren für präventives und repressives Vorgehen der Polizei ist nicht immer möglich, wenn sie gleichzeitig Aufgaben der Strafverfolgung und der Gefahrenabwehr wahrnimmt. Anders als bei der Frage nach dem Rechtsweg oder nach der Ermächtigungsgrundlage kann eine Handlung gebührenrechtlich nicht in zwei Handlungen aufgeteilt werden. ${ }^{79} \mathrm{Da}$ für die Kostenabrechnung insbesondere der Zeitaufwand maßgeblich ist, müßte die doppelfunktionale Maßnahme der Polizei zeitlich trennbar sein, was aber tatsächlich nicht geht. ${ }^{80}$

Ebensowenig ist ein rechnerisches Herausnehmen der strafrechtlichen Seite des Polizeieinsatzes durch Abzug von den polizeirechtlichen Kosten konsequent, da so strafrechtlich Verfolgte vor nur polizeiwidrigen Störern privilegiert würden. ${ }^{8_{1}}$ Eine spätere Heranziehung bei einem strafrechtlichen Freispurch würde die gesamte strafprozessuale Kostenregelung in Frage stellen. Ebenso würde eine spätere Anrechnung der polizeirechtlichen Gebühren auf die Strafverfahrenskosten die strafprozessuale Regelung unterlaufen. Sie könnte z. B. zu dem absurden Ergebnis führen, daß ein Straftäter nur deswegen Rechtsmittel einlegt, weil die schon von ihm bezahlten Polizeikosten auch noch die Kosten einer weiteren Strafgerichtsinstanz abdecken würden.

Der Fall liegt also bei Störern i. S. d. Polizeirechts anders als bei polizeikostenpflichtigen Großveranstaltern. Bei deren Kostenpflicht kann klarer zwischen dem Einsatz von Ordnungskräften und Strafverfolgungsbeamten (z. B. Kriminalpolizei) unterschieden werden. ${ }^{82}$

Die von Schenke geforderte völlige Selbständigkeit beider Regelungsmaterien ${ }^{83}$ führt dazu, daß der Betroffene für einmaliges staatliches Handeln zweimal zur Kasse gebeten wird. Dies widerspricht dem Kostendeckungsprinzip des Gebührenrechts.

78 Vgl. $\$ 46$ s StPO; Kleinknecht StPO, 3s. Aufl., $\$ 464$ a Rdn. 2.

79 Schilde (Fußnote 9), S. 90; m. V. a. Schwan Verw. Archiv 70 (1979), S. 109 ff.; anders VGH Mannheim (Fußnote 32), S. 9 f. (DÖV 1984 , S18f.)

80 Götz (Fußnote 32), S. 20.

81 Albrecht (Fußnote 65), S. 175

82 VGH Mannheim DVBl. 1981, 779.

83 Schenke (Fußnote 31), S. 1889 . 
Die Privilegierung von Strafverfolgten gegenüber gebührenpflichtigen "einfachen « Störern verstößt gegen den Gleichheitsgrundsatz. ${ }^{84}$

b) Der Landesgesetzgeber hatte für die Erstreckung der Gebührenpflicht auf Polizeimaßnahmen, die gegen Demonstranten und auch zum Zweck der Strafverfolgung durchgeführt werden, keine Gesetzgebungskompetenz. Dieser Gesichtspunkt kann wegen der grundrechtssichernden Funktion der Kompetenzordnung des Grundgesetzes $^{85}$ auch im Rahmen einer Anfechtungsklage eines einzelnen Betroffenen berücksichtigt werden. ${ }^{86}$

Der Bund hat mit den $\$ \$ 464 \mathrm{ff}$. StPO und den $\$ \$ 40 \mathrm{ff}$. GVG eindeutig von seiner Gesetzgebungskompetenz auf dem Gebiet des Strafrechts und des gerichtlichen Verfahrens nach Art. 74 Nr. 1 GG Gebrauch gemacht. Strafrecht i. S. d. Art. 74 Nr. 1 GG ist weit auszulegen und erfaßt auch das Ordnungswidrigkeitenrecht. ${ }^{87}$ Der Begriff "Strafrecht" ist vom Polizeirecht nicht nach der Rechtswegbestimmung abzugrenzen, vielmehr umfaßt er auch weite Bereiche des Polizeirechts, für die die Sperrwirkung des strafrechtlichen Kostenrechts gilt. ${ }^{88}$

Nach Art. 1 GG bricht Bundesrecht Landesrecht. Eine Anwendung der LVwVGKO in Fällen eines parallel geführten Strafverfahrens verstößt gegen Art. 31 GG. ${ }^{89}$

Wenn der Bundesgesetzgeber keine Kostenpflicht für polizeiliche Einsätze vorgesehen hat, bedeutet das nicht, daß die Gesetzgebungszuständigkeit der Länder wieder auflebt. Die Gesamtwürdigung des betreffenden Normenkomplex ergibt, daß der Bund den Zusammenhang abschließend regeln wollte und ergänzende Vorschriften ausgeschlossen sein sollten. ${ }^{9 \circ}$ Dies ist heute für das Strafprozeß-, das Gerichtskostenund das Ordnungswidrigkeitenrecht allgemein anerkannt. ${ }^{91}$ Etwaige Vorbehalte zugunsten der Länder sind ausdrücklich geregelt.

Es bedarf zur Lösung des Widerspruchs zwischen Bundes- und Länderregelung nicht der Heranziehung des Grundsatzes der Bundestreue. ${ }^{92}$ Das Land durfte keine Regelung treffen, die wie die LVwVGKO auch die Kostenpflicht im Fall einer Strafverfolgung vorsieht. Denkbar wäre höchstens eine verfassungskonforme Auslegung der LVwVGKO, die die Kostenerhebung in den genannten Fällen ausschließen würde. ${ }^{93}$

Die Unzuständigkeit des Landes für die sanktionsgleiche Kostenerhebung bei Demonstrationen resultiert auch aus dem faktischen Strafencharakter der Verordnung. Das Land kann sich nicht über die Hintertür des Polizeirechts Kompetenzen für Regelungen verschaffen, die nach Intention und Wirkung Strafvorschriften sind. ${ }^{94}$

Ähnliche Bedenken gegen die Landesregelung ergeben sich daraus, daß dem Bund nach Art. 74 Nr. 3 GG auch das Versammlungsrecht als konkurrierende Kompetenz

84 Kühling (Fußnote I), S. 317 ; Albrecht (Fußnote 65), S. 174; Schilde (Fußnote 9), S. 91; v. Brünneck (Fußnote 27), S. $277 \mathrm{f}$.

85 BVerfG NJW $198 \mathrm{I}, 33 \mathrm{I}$.

86 Schilde (Fußnote 9), S. 90.

87 Herzog (Fußnote 27), Art. 74 Rdn. 26; BVerfGE 27, I8.

88 Geulen (Fußnote 39), Anm. 3 r.

89 Albrecht (Fußnote 65), S. 175; Wilke, Gebührenrecht und Grundgesetz, 1973, S. 179 f.; Schilde (Fußnote 9), S. 90.

90 Vgl. BVerfGE 32, 327; Geulen (Fußnote 39), S. 95; Schilde (Fußnote 9), S. 89.

9 I Schäfer, in: Löwe/Rosenberg/Schäfer, StPO, vor $\$ 46_{4}$ Rdn. $2 \mathrm{ff}$.

92 So Kühling (Fußnote I), S. 317 , dazu Geulen (Fußnote 39), S. 97; Schilde (Fußnote 9), S. 91; Schenke (Fußnote 3I), S. I889.

93 Schilde (Fußnote 9), S. 90; Albrecht (Fußnote 65), S. 175; Wilke (Fußnote 89), S. 179 f.; v. Brünneck (Fußnote 27), S. 279.

94 v. Brünneck (Fußnote 27 ), S. 278. 
obliegt. Das Versammlungsrecht geht als lex specialis dem allgemeinen Polizeirecht vor. Die Gesamtwürdigung des Komplexes Versammlungsrecht ergibt, daß der Bund diese Materie zumindest insoweit abschließend regeln wollte, daß den Ländern keine Regelungszuständigkeit für die Erhebung von Einsatzkosten der Polizei offen bleibt. 95 Geulen wies darauf hin, daß die Sperrwirkung des Versammlungsgesetzes auch unfriedliche Versammlungen umfaßt, da auch diese dem Versammlungsgesetz unterfallen. ${ }^{96}$

\section{Ermächtigungsgrundlage}

a) Bisweilen wird in Frage gestellt, ob Kosten für Polizeieinsätze überhaupt nach Verwaltungskostenrecht geltend gemacht werden dürfen. ${ }^{97}$ Bei Fehlen eines speziellen Kostentatbestands im Polizeirecht dürfe nicht auf die Normen des allgemeinen Gebührenrechts zurückgegriffen werden, da das Polizeirecht als lex specialis abschließend sei. Anderenfalls könnte jede Amtshandlung der Polizei über allgemeines Gebührenrecht kostenpflichtig gemacht werden. Dem wird entgegengesetzt, daß die Frage, an welcher Stelle und in welchem Zusammenhang eine gesetzliche Ermächtigungsgrundlage statuiert wird, eine reine Frage der Gesetzestechnik bzw. -systematik sei. ${ }^{98}$ Man müsse davon ausgehen, daß der Landesgesetzgeber in den $\$ \$ 79,80$ PolG keine abschließende Regelung treffen wollte. In jedem Fall wäre eine solche Regelung durch die zeitlich spätere Verabschiedung des Verwaltungsvollstreckungsgesetzes korrigiert worden, wenn darin tatsächlich eine Ermächtigung zum Erlaß einer Verordnung über den Ersatz von Polizeikosten enthalten ist.

Folgt man der oben genannten Ansicht nicht, so kommt $\$ 3$ I III LVwVG als Gesetzesgrundlage in Frage. Die Erhebung von Polizeikosten von Privatpersonen stellt einen Eingriff in Freiheit und Eigentum des Bürgers dar und unterliegt deshalb dem Vorbehalt des Gesetzes (Art. 20 III GG), d. h. sie bedarf der Ermächtigung in einem Gesetz. Wegen der Nähe zu den Grundrechten aus Art. 5 und 8 GG muß die Polizeikostenpflicht als eine "wesentliche Grundentscheidung«i.S.d. Rechtsprechung des BVerfGs angesehen werden, die ausdrücklich vom Gesetzgeber getroffen worden sein muß.99

b) Die Ermächtigungsnorm der Verordnung, $\$ 3$ I III LVwVGKO, ist wegen ungenügender Bestimmtheit keine ausreichende gesetzliche Grundlage. ${ }^{100}$ Das Bestimmtheitserfordernis ist Ergänzung und Konkretisierung des Demokratie- und Rechtsstaatsprinzips - Prinzipien, welche ihre Normierung in Art. 20 und 28 I I GG gefunden haben. ${ }^{\text {IOI }}$ Art. 80 I 2 GG und Art. 6I I 2 badenwürttembergische LandVerf fordern von einem Ermächtigungsgesetz, daß dieses Inhalt, Zweck und Ausmaß der Rechtsverordnung bestimmt.

Das Erfordernis hinreichender Bestimmtheit hängt von der Grundrechtsrelevanz der Norm ab, zu der ermächtigt wird. Greift die Regelung erheblich in die Rechtsstellung des Betroffenen ein, so müssen höhere Anforderungen an den Bestimmtheitsgrad der Ermächtigung gestellt werden, als wenn es sich um einen Regelungsbereich

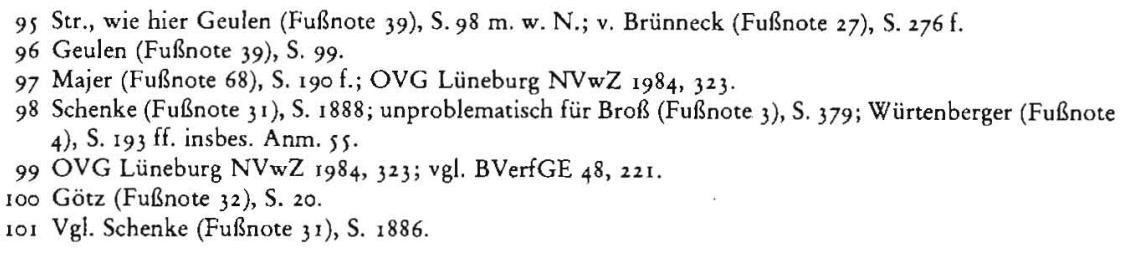


handelt, der die Grundrechtsausübung weniger tangiert. ${ }^{102}$ Bei Eingriffsgesetzen müssen besonders strenge Anforderungen an die inhaltliche Bestimmtheit gestellt werden. ${ }^{103}$

Die nötige Bestimmtheit fehlt, wenn nicht mehr vorhergesehen werden kann, in welchen Fällen und mit welcher Tendenz von einer Ermächtigungsnorm Gebrauch gemacht wird und welchen Inhalt die auf Grund der Ermächtigung erlassene Verordnung haben kann. ${ }^{104}$ Der Gesetzgeber muß die Grenzen einer Regelung festsetzen und angeben, welchem Ziel die Regelung dienen soll.

Die LVwVGKO besitzt durch die Anwendbarkeit auf friedliche Demonstrationen besondere Grundrechtsrelevanz, so $\mathrm{da}$ ß besonders hohe Anforderungen an den Bestimmtheitsgrad gestellt werden müssen.

Das BVerfG präzisiert die Erfordernisse an die Bestimmtheit einer Ermächtigungsnorm zum Erlaß einer Gebührenregelung wie folgt: Aus ihr muß etwas über den zu regelnden Gegenstand zum Ausdruck kommen. Für die nötige Konkretisierung reichen das Kostendeckungs- und das Äquivalenzprinzip nicht aus. Auch bei Beachtung dieser Grundsätze sind Gebührenordnungen denkbar, die völlig verschieden sind und die den Staatsbürger in verschiedener Weise belasten. Deshalb muß die Ermächtigung selbst ein Minimum an materieller Regelung enthalten, die dem Verordnungsgeber als »Rahmen« und »Programm« dienen soll und kann. Sie muß ihm auch Grenzen setzen. ${ }^{105}$

Im Gegensatz zu den dargelegten Erfordernissen überläßt $\$ 31$ III LVwVG die Gestaltung in vollem Umfang dem Verordnungsgeber. Dieser kann bestimmen, welche Akte die Gebührenpflicht auslösen, wie hoch die Gebühren sein dürfen, ob und welcher Ermessensspielraum der festsetzenden Behörde zugestanden wird. Nach dem Gesetz erstreckt sich der Anwendungsbereich der Kostenerhebung unterschiedslos auf alle Vollstreckungshandlungen. Es bleibt z. B. die wesentliche Frage vollständig offen, ob und inwieweit für die Anwendung unmittelbaren $Z_{\text {wanges }}$ Gebühren erhoben werden dürfen. Angesichts der Unvorhersehbarkeit des Ausmaßes eines polizeilichen Einsatzes und damit des Ausmaßes der Gebühr konnte deren Regelung nicht dem Verordnungsgeber überlassen werden. ${ }^{106}$

Die Ermächtigung ist so unbestimmt, daß nicht mehr vorhergesehen werden kann, mit welcher Tendenz von ihr Gebrauch gemacht wird und verstößt daher gegen Art. 80 I 2 GG und Art. 6I I 2 Baden-Württembergische LandVerf. ${ }^{107}$

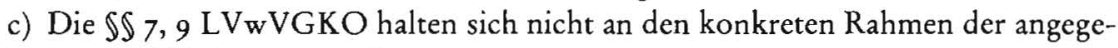
benen Ermächtigungsgrundlage.

Das LVwVG läßt als Zweck der Kostenerhebung nur die Finanzierung der öffentlichen Verwaltung erkennen. Mit der neuen Kostenverordnung ist unbestrittenermaßen auch die Disziplinierung von Störern beabsichtigt. Dies kann nicht mehr durch die gesetzliche Regelung des LVwVG abgedeckt werden. ${ }^{\text {ros }}$

Nach $\rrbracket_{3}$ I III LVwVG sind die Gebührensätze nach der Bedeutung der Amtshandlung für den Pflichtigen zu bemessen. Anders als bei Polizeieinsätzen für private Veranstaltungen geschieht die Anwendung unmittelbaren Zwanges immer im öffentlichen Interesse. Tatsächlich hat der Betroffene keinerlei Vorteile von der Zwangsanwendung, diese ist für ihn unerwünscht. Als Rechtfertigung für die

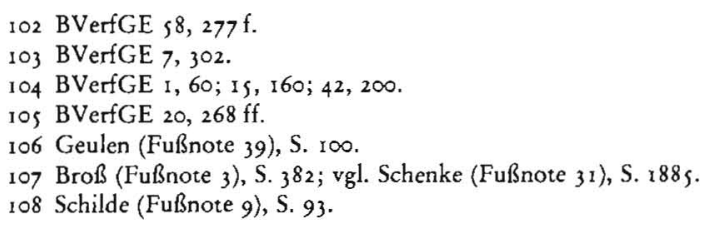


Kostenerhebung trotz fehlenden Individualinteresses kann nicht $₫ 35$ IV PolG herangezogen werden. ${ }^{109} \$ 35$ IV PolG wird nicht als Ermächtigungsnorm für die Verordnung genannt und ist hierzu auch nicht geeignet. $\$_{35}$ IV PolG verweist auf $\mathbb{3}_{3} \mathrm{I}$ LVwVG. Die polizeirechtliche Regelung kann den Anwendungsbereich des $\mathbb{3} \mathrm{I}$ LVwVG nicht erweitern.

Von $\$ 3_{1}$ III LVwVG ebenso wenig gedeckt ist die in $\$ 7 \mathrm{LVwVGKO}$ eingeführte Zeitgebühr. Zulässig sind nach Satz 2 nur Fest- und Rahmengebühren. Die Zeitgebühr ist der Sache nach eine Form des Aufwendungsersatzes, die nach völlig anderen Kriterien als eine Fest- oder Rahmengebühr berechnet wird. ${ }^{10}$ Satz 3 eröffnet nicht die Möglichkeit zu einer über Satz 2 hinausgehenden Differenzierung, sondern legt die Bemessung der Gebühren nach Satz 2 näher fest. ${ }^{\text {"I }}$

Schließlich ist fraglich, ob der Auslagenbegriff des $\$ 31$ III LVwVG zuläßt, daß der eigene Personal- und Materialaufwand der Polizei berechnet wird ( $\$ 8 \mathrm{LVwVGKO}$ ). Auslagen sind nur die Aufwendungen, die die Verwaltung zur Vornahme der Amtshandlung an Dritte macht. ${ }^{12}$

\section{Sonstige Rechtsprableme}

a) Voraussetzung jeder zulässigen Gebührenerhebung ist die sogenannte individuelle Zurechenbarkeit der staatlichen Leistung. ${ }^{113}$ Eine solche Einzelzuordnung ist bei einer Demonstration, einem politischen Kollektivverhalten, praktisch unmöglich, Der Polizeiaufwand bemißt sich nach allgemein politischen Stimmungen, die für eine Demonstration prognostiziert werden, und nach Kriterien, die gerade in deren kollektivem Charakter wurzeln. Der Polizeiaufwand ist nicht individuell zurechenbar, auch dann nicht, wenn man die Kosten »angemessen verteilt«. Dies gilt natürlich um so mehr für die gesamtschuldnerische Haftung. ${ }^{14}$

In bezug auf die Zurechenbarkeit entstehen auch auf der subjektiven Seite Probleme. Unbestritten ist, daß die Kostenpflicht nicht nach den strengen strafrechtlichen Schuldprinzipien behandelt wird. Störer ist nicht nur, wer vorsätzlich handelt, sondern jeder, der objektiv eine Störung für die öffentliche Sicherheit und Ordnung hervorruft. Um eine Zurechnung begründen zu können, ist zumindest ein gewisser Grad an Fahrlässigkeit bei der Verfügungsmißachtung erforderlich. Hiervon ist aber in der LVwVGKO keine Rede.

Hinzukommt, daß, anders als bei individuellen Polizeiverfügungen, es in einer Demonstration praktisch unmöglich sein kann, die subjektiven Voraussetzungen für die Kostenpflicht festzustellen.

b) Nicht nur bei der Ermächtigungsnorm, auch bei der Rechtsverordnung selbst ist nach Art. 20, 28 I I GG von den verwendeten Rechtsbegriffen hinreichende Bestimmtheit zu fordern. " Gesetzesbegriffe einer einheitlichen Handhabung und klaren Konkretisierung zuführen. Der Bürger muß klar aus der Rechtsverordnung entnehmen können, wel-

109 So Herzog, in: Der Spiegel Nr. 52/82, S. 30.

I10 Götz (Fußnote 32), S. 22; Kilian (Fußnote 32), S. 53.

II A. A. VGH Mannheim B. v. 26. 3. 1984 (Fußnote 32), S. I2; DÖV 1984, 517 insoweit nicht abgedruckt.

112 Götz (Fußnote 32), S. 23; Kilian (Fußnote 32), S. 54.

I 3 Schenke (Fußnote 3r), S. I 890 ; Götz (Fußnote 32), S. 19; v. Brünneck (Fußnote 27), S. 276

II Schilde (Fußnote 9), S. 92.

I Is Kühling (Fußnote I), S. 3 16; Götz (Fußnote 68), S. 172. 
che rechtlichen Konsequenzen sein Verhalten haben wird. ${ }^{116}$ Diesen Erfordernissen genügt die Kostenverordnung nicht.

Dies gilt zunächst für den für die Kostenhöhe wesentlichen Begriff der mangemessenen Verteilung" der Gebühren ( $\$$ g LVwVGKO). Unklar bestimmt ist, ob die Kosten auf alle Demonstrationsteilnehmer, auf alle Personen, gegen die die Polizeiverfügung erging und die ihr nicht sofort folgten (so wohl die Praxis), auf alle, gegen die unmittelbarer $Z$ wang angewendet wurde, oder auf die, deren Personalien festgestellt wurden, verteilt werden.

Gebühren werden erhoben für »jeden bei der Anwendung unmittelbaren $Z$ wanges eingesetzten Bediensteten « ( $\$ 7$ II LVwVGKO). Dies könnte bedeuten: alle tatsächlich eingesetzten Beamten des Gesamteinsatzes (so vorwiegende Praxis), die hierfür nötigen Beamten, die bei einer konkreten Räumung eingesetzten Beamten ${ }^{17}$, die für die Räumung nötige Mindestzahl, nur die Beamten, die gegen den Betroffenen tatsächlich Zwang anwendeten. ${ }^{1{ }^{18}}$ Ungeklärt bleibt, ob bei einer ex-ante-Betrachtung der "Erforderlichkeit von Polizeikräften« von dem unbedingt erforderlichen Aufwand auszugehen ist oder, was wohl der Ansicht des VGH Mannheim entsprich ${ }^{\mathrm{I}}{ }^{\mathrm{I}}$, ob alles angerechnet werden kann, was nicht offensichtlich entbehrlich und unsachlich motiviert ist. ${ }^{120}$

Nach $₫>$ LVwVGKO beträgt die Gebühr je angefangene Stunde pro Bediensteten $38 \mathrm{DM}$. Unbestimmt ist, ob die Stunden des gesamten Einsatzes gerechnet werden, mit oder ohne Anfahrtszeit (so wohl PD Sigmaringen), oder ob nur die Zeit der tatsächlichen Zwangsanwendung zählt (so PD Offenburg, LPD Stuttgart). ${ }^{\text {r2t }}$

Völlig unbestimmt ist weiterhin der Zeitpunkt, an dem die Gebührenpflicht entsteht. Ist hierfür die Polizeiverfügung maßgeblich, der Zeitpunkt, an dem sich die Polizeibeamten an Ort und Stelle befinden, oder der Moment, in dem sich ein durchschnittlicher oder der konkrete Mensch der Verfügung frühestens fügen konnte, oder erst der Beginn der Zwangsanwendung selbst?

Bei all diesen Punkten wirft die neue LVwVGKO mehr Fragen auf, als sie beantwortet. Wegen der Unbestimmtheit der Verordnungsregelung wird über die richtige Berechnungsart nie Klarheit hergestellt werden können. Auch diese Unbestimmtheit führt zur Rechtswidrigkeit der Verordnung.

c) Die Praxis wird noch eine Vielzahl weiterer Unsicherheiten bei der Anwendung der neuen $L V_{w}$ VGKO ergeben, die nur schwer mit dem Rechtsstaatsprinzip vereinbar sind. So scheint sich der Anwendungsbereich der Neuregelung fast ausschließlich auf politische Demonstrationen in Form gewaltfreier Aktionen zu beschränken, während für die »alltägliche« Anwendung unmittelbaren Zwanges keine Gebühren gefordert werden - eine Praxis, die nicht gerade ein Beispiel von Gleichbehandlung ist. Es besteht auch die Gefahr, daß sich die Polizei in ihrem Auftreten bei Demonstrationen von der Kostenverordnung leiten läßt. Das kann mit dem Einsetzen vieler Beamten bei mißliebigen Demonstrationen beginnen, deren Kosten eventuell abgewälzt werden können, und sogar dahin führen, daß die Polizei von sich aus Demonstranten provoziert, um diese zu einem einen Einsatz erfordernden Verhalten zu bringen, das zur Rechtfertigung der Kostenerhebung nötig ist. ${ }^{122}$

Auf jeden Fall ist die Polizei durch die Kostenverordnung zu einer aufwendigen

I16 Leibholz/Rinck, Grundgesetz, Stand I982, Art. 20 Rdn. 24.

117 So Herzog, in: Der Spiegel Nr. 52/82, S. 29.

118 So Schenke (Fußnote 31), S. 1889 ; dazu Schilde (Fußnote 9), S. 90; Kilian (Fußnote 32), S. 54.

I 9 VGH Mannheim DÖV 1981, 805.

$120 \mathrm{Vgl}$. hierzu Schenke (Fußnote 31), S. 1889.

I2I Götz (Fußnote 32), S. 22.

122 Gerhard, NDR-Juristenstammtisch (Fußnote 74), S. 9. 
Beweisführung gezwungen (mehr Beamte, Filmen, Fotografieren), die einerseits den Vollzugsdienst belastet, andererseits zu einem verstärkten Polizeieinsatz führt. Dies wiederum hat negative Auswirkungen auf die Bereitschaft von Bürgern, sich an Demonstrationen zu beteiligen.

d) Nur angeschnitten werden kann an dieser Stelle die Frage, ob der Kostenersatz beim unmittelbaren Zwang zu den »öffentlichen Abgaben und Kosten « i. S. d. $\$ 80$ II Nr. I VwGO gerechnet wird. Diese baden-württembergische Verwaltungspraxis wurde vom VGH Mannheim als rechtmäßig erklärt. ${ }^{123}$ Der VGH beruft sich dabei auf den Willen des Landesgesetzgebers, der den Polizeikostenersatz gemeinsam mit sonstigen Verwaltungsgebühren regelte. Kosten des Verwaltungsvollstreckungsverfahrens seien denen des Verwaltungs- und Vorverfahrens gleichzustellen. Dem hält das VG Freiburg richtigerweise entgegen, $\$ 80$ II Nr. I VwGO sei wegen seines Ausnahmecharakters einengend auszulegen. ${ }^{124}$ Der Kostenersatz ist dem Grund nach etwas völlig anderes als eine Verwaltungsgebühr. Er ist nicht Gegenleistung für eine vorteilhafte Amtshandlung, sondern ist Erstattung für Aufwendungen der öffentlichen Hand, deren Grund und Höhe sich nicht pauschal, sondern nach den jeweiligen Umständen des Einzelfalls bestimmt. ${ }^{\text {I2s }}$

Es liegt nun an den Gerichten, ob der Ersatz von Polizeikosten bei Demonstrationen nur eine Episode war oder ob eine weitere Einschränkung der Demonstrationsfreiheit ihren Segen erhält. Das OVG Lüneburg hat hier richtige Weichen gestellt.

Thilo Weichert

123 VGH Mannheim B. v. 26. 3. 1984 (Fußnote 32), S. 3-7; DÖV 1984, 517f.

124 VG Freiburg B. v. 7. 10. $1983,2 \mathrm{~K} 226 / 83$.

I25 Götz (Fußnote 32), S. 14 f. m. w. N.

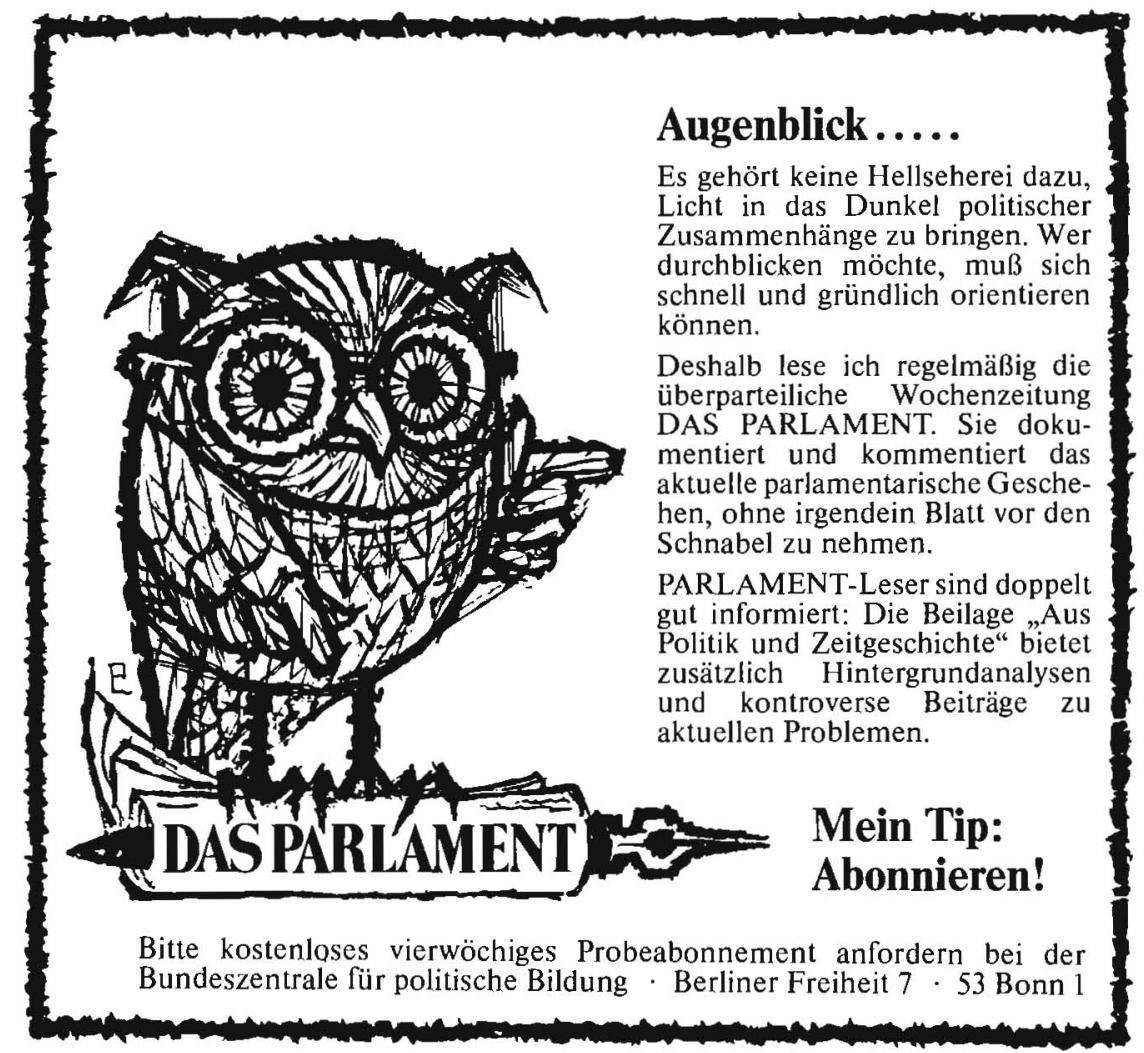

